\title{
MINIMAL SETS: AN INTRODUCTION TO TOPOLOGICAL DYNAMICS ${ }^{1}$
}

\author{
W. H. GOTTSCHALK
}

The notion of minimal set is centrally located in topological dynamics. Topological dynamics may be defined as the study of transformation groups with respect to those properties, wholly or largely topological in nature, whose prototype occurred in classical dynamics.

Henri Poincare was the first to introduce topological notions and methods in dynamics, that is, the study of ordinary differential equations. G. D. Birkhoff was the first to undertake the systematic development of topological dynamics, indicating its essentially abstract character and making fundamental contributions. Birkhoff's first paper on the subject appeared in 1912 [6, pp. 654-672]; Birkhoff's paper contains the first definition of minimal sets, some theorems about them, and some examples of them. Most of Birkhoff's work in topological dynamics from the point of view of general theory is to be found in Chapter 7 of his Colloquium volume Dynamical systems published in 1927 [5]. The Colloquium volume Analytical topology by G. T. Whyburn [27], published in 1942, contains related developments in its Chapter 12. The Russian book, Qualitative theory of differential equations by Nemyckil and Stepanov [22], first edition in 1947 and second edition in 1949, contains a chapter devoted to topological dynamics; an English translation of this book has recently been announced by Princeton Press. The treatments of topological dynamics in the above books are all from the points of view of a single transformation or a one-parameter group of transformations. The Colloquium volume Topological dynamics by Hedlund and myself [14], published in 1955, is concerned mainly with general transformation groups.

A topological transformation group, or transformation group for short, is defined to be an ordered triple $(X, T, \pi)$ such that the following axioms are satisfied:

(A0) (Stipulative AXIOM). $X$ is a topological space, called the phase space; $T$ is a topological group, called the phase group; and $\pi$ is a map of the cartesian product $X \times T$ into $X$, called the phase map.

An address delivered before the Washington meeting of the Society on October 26, 1957, by invitation of the Committee to Select Hour Speakers for Eastern Sectional Meetings; received by the editors April 10, 1958.

1 This address was prepared while the author was under contract No. AF 18(600)1116 of the Air Force Office of Scientific Research. Reproduction in whole or in part is permitted for any purpose of the United States Government. 
[Let us for the moment use the multiplicative notation for the phase group $T$, let $e$ denote the identity element of $T$, and let the value of $\pi$ at the point $(x, t)$ of $X \times T$ be denoted by $x t$.]

(A1) (Identity AXIom), $x e=x$ for all $x \in X$.

(A2) (Homomorphism AXIOM). $(x t) s=x(t s)$ for all $x \in X$ and all $t, s \in T$.

(A3) (Continuity AXIOM), $\pi$ is continuous.

Consider a given transformation group $(X, T, \pi)$. The phase map $(x, t) \rightarrow x t$ determines two kinds of maps when one of the variables $x, t$ is replaced by a constant. Thus, for fixed $t \in T$, the map $x \rightarrow x t$ is a homeomorphism $\pi^{t}$ of the phase space $X$ onto itself which is called a transition. Again, for fixed $x \in X$, the map $t \rightarrow x t$ is a continuous map $\pi_{x}$ of the phase group $T$ into the phase space $X$ which is called a motion. The set of all transitions is a group of homeomorphisms of the phase space $X$ onto itself such that the transition $\pi^{e}$ induced by the identity element $e$ of $T$ is the identity homeomorphism of the phase space $X$ and such that the map $t \rightarrow \pi^{t}$ is a group homomorphism. Conversely, a group of homeomorphisms of $X$ onto itself, suitably topologized, gives rise to a transformation group.

An intrinsic property of the transformation group $(X, T, \pi)$ is defined to be a property which is describable in terms of the topology of the phase space $X$, the topology and group structure of the phase group $T$, and the phase map $\pi$. Topological dynamics is concerned with intrinsic properties of transformation groups with particular reference to those properties which first arose in classical dynamics.

Among the examples which have been studied in the past, the most frequently occurring phase groups are the additive group $\mathfrak{g}$ of integers with the discrete topology and the additive group $R$ of real numbers with its usual topology. In either case, the transformation group is called a flow. If the phase group $T$ is $\mathscr{G}$, then the transformation group is called a discrete flow. If the phase group $T$ is $R$, then the transformation group is called a continuous flow. Here the word "continuous" apparently refers to the "real number continuum".

To see how transformation groups in the role of continuous flows appeared in classical dynamics, consider a system of $n$ first order ordinary differential equations of the form

$$
\frac{d x_{i}}{d t}=f_{i}\left(x_{1}, \cdots, x_{n}\right) \quad(i=1, \cdots, n)
$$

where the functions $f_{i}$ may be defined on a region $X$ of euclidean $n$ - 
space or they may be defined locally throughout an $n$-dimensional differentiable manifold $X$. Impose enough conditions to insure the existence, uniqueness, and continuity of solutions for all real values of the "time" $t$ and through all points of $X$. Then there exists exactly one continuous flow in $X$ whose motions are the solutions of the system of differential equations. To illustrate with a very simple example, the continuous flow in the plane determined by the system

$$
\frac{d x_{1}}{d t}=x_{1}, \quad \frac{d x_{2}}{d t}=-x_{2}
$$

has as phase map $\pi\left(\left(x_{1}, x_{2}\right), t\right)=\left(x_{1} e^{t}, x_{2} e^{-t}\right)$.

A dynamical system may be defined as a continuous flow in a differentiable manifold induced by an autonomous system of first order ordinary differential equations, as above. Of course, every dynamical system is a continuous flow. To what extent and under what conditions a continuous flow is a dynamical system appears to be a largely unsolved problem. Let me say that this usage of the term "dynamical system" is not at all universal. More likely than not it is often used as synonymous with "continuous flow" or even "discrete flow."

The two kinds of flows, discrete and continuous, are closely related. For example, a continuous flow determines many discrete flows by taking cyclic subgroups of $R$. Conversely, a discrete flow determines a continuous flow when the phase space $X$ is extended to the cartesian product of $X$ and the closed unit interval, and the bases of the cylinder thus obtained are identified according to the transition induced by the integer 1 , that is, $(x, 1)$ and $(x 1,0)$ are identified. The problem of imbedding a discrete flow in a continuous flow without alteration of the phase space appears to be largely unsolved.

A discrete flow determines and is determined by the transition induced by the integer 1 . Thus, we may redefine a discrete flow more economically as an ordered pair $(X, \phi)$ where $X$ is a topological space and $\phi$ is a homeomorphism of $X$ onto $X$. For the sake of simplicity, I wish to confine most of my remaining remarks to flows, and in particular to discrete flows. Moreover, the phase space will be assumed to be compact metric for the same reason. It is to be pointed out that the present theory of topological dynamics contains results on transformation groups acting upon topological spaces and uniform spaces which specialize to give most of the known facts about flows on metric spaces.

Let $X$ be a nonvacuous compact metric space with metric $\rho$ and let $\phi$ be a homeomorphism of $X$ onto $X$. 
The phase space $X$ is nonvacuous closed and invariant, that is, $X \phi=X$. In case $X$ has these properties minimally it is said that $X$ is a minimal set. The definition may be phrased for a subset of $X$. A subset $M$ of $X$ is said to be minimal under $\phi$ provided that $M$ is nonvacuous closed and invariant, that is $M \phi=M$, and no proper subset of $M$ has all these properties. Minimal sets may be characterized in various ways. If $x \in X$, then the orbit of $x$, denoted $O(x)$, is defined to be $\left\{x \phi^{n} \mid n \in \mathscr{g}\right\}$ where $\mathscr{I}$ is the set of all integers; and the orbit-closure of $x$, denoted $\bar{O}(x)$, is defined to be the closure of the orbit $O(x)$ of $x$. A subset $M$ of $X$ is minimal if and only if $M$ is nonvacuous and $M$ is the orbit-closure of each of its points.

By the axiom of choice, there always exists at least one minimal subset of $X[14$, p. 15].

Now recursive properties enter into consideration. A point $x$ of the phase space is said to be almost periodic under $\phi$ and $\phi$ is said to be almost periodic at $x$ provided that if $U$ is a neighborhood of $x$, then there exists a relatively dense subset $A$ of $\mathscr{g}$ such that $x \phi^{n} \in U$ for all $n \in A$. A subset $A$ of $\mathfrak{g}$ is called relatively dense in case the gaps of $A$ are bounded. Another way of saying the same thing is that $\mathfrak{g}=A+K=\{a+k \mid a \in A \& k \in K\}$ for some finite subset $K$ of $g$. We may call the set $K$ a bond of the set $A$. A periodic point is almost periodic, but not conversely. It has been remarked that a periodic point returns to itself every hour on the hour; but an almost periodic point returns to a neighborhood every hour within the hour.

The basic connection between minimal sets and almost periodic points is this: The orbit-closure of a point $x$ is minimal if and only if the point $x$ is almost periodic [14, p. 31]. Consequently, every point of a minimal set is almost periodic, and there always exists at least one almost periodic point. This, of course, is in strong contrast to the situation with respect to periodic points.

Since different minimal subsets of $X$ are necessarily disjoint, we may conclude that the class of all orbit-closures is a partition of the phase space if and only if $\phi$ is pointwise almost periodic, that is to say, $\phi$ is almost periodic at each point of $X$. The partition of orbitclosures, when it exists, is necessarily star-open in the sense that the saturation or star of every open set is again open. In a compact metric space, which we are here considering, this is equivalent to lower semicontinuity in another terminology. It is however not always the case that the partition of orbit-closures is star-closed in the sense that the star of every closed set is itself closed, or equivalently, upper semicontinuous. This condition may be characterized by the following recursive property. The map $\phi$ is said to be weakly almost periodic on 
$X$ and the phase space $X$ is said to be be weakly almost periodic under $\phi$ provided that if $\epsilon$ is a positive real number, then there exists a finite subset $K_{\mathrm{e}}$ of $\mathfrak{g}$ such that $x \in X$ implies the existence of a (necessarily relatively dense) subset $A_{x}$ of $\mathscr{g}$ such that $\mathfrak{g}=A_{x}+K_{\mathfrak{\epsilon}}$ and $x \phi^{n} \in N_{\epsilon}(x)$ for all $n \in A_{x}$. Here the bond $K_{\epsilon}$ depends only upon $\epsilon$, but the individual sets of return $A_{x}$ depend also upon $x$. To summarize, the class of all orbit-closures is a star-closed partition of the phase space if and only if the map $\phi$ is weakly almost periodic [14, p. 34]. In particular, $\phi$ is weakly almost periodic on each minimal set.

We are now in the middle of the spectrum of recursive properties. A weaker recursive property than almost periodic may be mentioned first of all. A point $x$ of the phase space is said to be recurrent provided that if $U$ is a neighborhood of $x$, then there exists an extensive subset $A$ of $g$ such that $x \phi^{n} \in U$ for all $n \in A$. Here "extensive" means containing a sequence diverging to $-\infty$ and a sequence diverging to $+\infty$. In other words a point is recurrent provided that it returns (or recurs) to a neighborhood infinitely often in the past and infinitely often in the future. The term recurrent as it is here used coincides with its meaning in the Poincare Recurrence Theorem (1899), which seems to be one of the earliest appearances in the literature of a theorem on a recursive property different from periodic. In general, pointwise recurrent does not imply pointwise almost periodic [12]. However, if the phase space is also zero-dimensional, then pointwise recurrent implies weakly almost periodic $[14$, p. 65].

Corresponding to any recursive property there is also the property of regionally recursive. For example, $\phi$ is regionally recurrent provided that if $x \in X$ and if $U$ is a neighborhood of $x$, then there exists an extensive subset $A$ of $\mathfrak{g}$ such that $U \cap U \phi^{n} \neq \varnothing$ for every $n \in A$. The center of a discrete flow $(X, \phi)$ is defined to the greatest invariant subset on which $\phi$ is regionally recurrent. The center is also characterized as the closure of the set of recurrent points. Since every almost periodic point is also recurrent, the center is necessarily nonvacuous. The center has the property that the relative sojourn of every point of the phase space in a neighborhood of the center is equal to unity. If the flow has an invariant measure which is positive and finite for nonvacuous open sets, then the flow is regionally recurrent and the center is the entire phase space. This is the case for conservative dynamical systems $[14 ; 22]$.

Let us examine a few stronger recursive properties. The map $\phi$ is said to be almost periodic on $X$ and the phase space $X$ is said to be almost periodic under $\phi$ provided that if $\epsilon>0$, then there exists a relatively dense subset $A$ of $\mathscr{g}$ such that $x \phi^{n} \in N_{\epsilon}(x)$ for all $x \in X$ and 
all $n \in A$. Almost periodic implies weakly almost periodic which in turn implies pointwise almost periodic; no converse holds. The almost periodicity of the map $\phi$ may be characterized as follows. These four statements are pairwise equivalent: (1) $\phi$ is almost periodic; (2) the set of powers of $\phi$, namely $\left\{\phi^{n} \mid n \in \mathcal{G}\right\}$, is equicontinuous; (3) the set of powers of $\phi$ has compact closure where the ambient space is the group of all homeomorphisms of $X$ onto $X$ with the usual topology; (4) there exists a compatible metric of $X$ which makes $\phi$ an isometry $[14$, p. 37].

A still stronger recursive property is regularly almost periodic (G. T. Whyburn 1942; P. A. Smith 1941 related) [27; 24]. This is like almost periodic except that the set of return $A$ is taken to be a nontrivial subgroup of $g$. We have then the properties: regularly almost periodic point, pointwise regularly almost periodic map, and regularly almost periodic map. We mention a few results in this context. The map $\phi$ is regularly almost periodic if and only if $\phi$ is both pointwise regularly almost periodic and weakly almost periodic. If $\phi$ is pointwise regularly almost periodic, then every orbit-closure is a zero-dimensional regularly almost periodic minimal set $[14$, p. 49f.]. That a regularly almost periodic homeomorphism on a manifold is necessarily periodic has been conjectured by P. A. Smith (1941) [24], but so far has been proved only for the two-dimensional case [for example, 14, p. 56].

By definition, an inheritance theorem is a theorem of the form " $\phi$ has property $P$ if and only if $\phi^{n}$ has the property $P$ " where $n$ is a preassigned nonzero integer. The inheritance theorem holds for recurrent and almost periodic points as well as for certain other recursive properties of a point. Actually, a very general inheritance theorem can be proved which yields most known results for recursive points $[14$, p. 26]. No general inheritance theorem is known for such properties as regionally recurrent and weakly almost periodic although they too inherit, at least under certain conditions $[14, \mathrm{pp} .67$ and 35 ].

In this connection we may mention the fact that a connected minimal set under $\phi$ is also minimal under $\phi^{n}(n \neq 0)[14$, p. 16].

Two discrete flows $(X, \phi)$ and $(Y, \psi)$ are said to be isomorphic provided there exists a homeomorphism $h$ of $X$ onto $Y$ such that $\phi h=h \psi$.

Let us consider now the problems: (1) (Construction problem.) To construct all minimal sets systematically; (2) (Classification problem.) To classify all minimal sets according to isomorphism type. At the present, only partial answers can be given to these questions, even for discrete flows on compact metric spaces. 
In the spectrum of isomorphism types of minimal sets, those which are almost periodic seem to constitute an extreme case, for the following reason. A monothetic group is by definition a compact group which contains a dense cyclic subgroup, a generator of a dense cyclic subgroup being called a generator of the monothetic group. Now the almost periodic minimal sets under discrete flows are coextensive with the monothetic groups in the following sense. If $X$ is a monothetic group with generator $a$, then $X$ is a minimal set under the homeomorphism $\phi$ of $X$ onto $X$ defined by $x \rightarrow x a$. Conversely, if $(X, \phi)$ is an almost periodic minimal set, then there exists a group structure in $X$ which makes $X$ a monothetic group and such that the map $\phi$ is translation by a generator $a$, that is $x \phi=x a$ for all $x \in X$ $[14$, p. 39]. Since the monothetic groups can be constructed in their entirety as the character groups of the subgroups of the discrete circle group (Anzai and Kakutani 1943) [2], the construction problem for almost periodic minimal sets under discrete flows has a reasonably definite answer. It is not clear to me whether the corresponding classification problem can also be answered by the present theory of topological groups. It is known (Halmos and Samelson 1942; Anzai and Kakutani 1943) $[15 ; 2]$ that every compact connected separable abelian group is monothetic. For example, the $n$-toral groups $K^{n}$ where $n$ is a positive integer and the infinite toral group $K^{\mathfrak{g}}$ are monothetic.

The $n$-adic groups are monothetic and are topologically the Cantor discontinuum. Let us describe geometrically a minimal set given by the dyadic group and a generator. A similar construction is available for the general case.

If $E$ is the disjoint union of segments (meaning closed line segments) $E_{1}, \cdots, E_{r}$, let $E^{*}$ denote the union of segments obtained by deleting the open middle third of each of the segments $E_{1}, \cdots, E_{r}$. Let $S$ be a segment of length 1 . Define $X_{0}, X_{1}, \ldots$ inductively as follows: $X_{0}=S, X_{n+1}=X_{n}^{*}(n=0,1,2, \cdots)$. Define $X=\bigcap_{n=0}^{+\infty} X_{n}$. The space $X$ is the Cantor discontinuum. Denote the $2^{n}$ disjoint segments which make up $X_{n}(n=0,1,2, \cdots)$ by $S(n, m)(0 \leqq m$ $\left.\leqq 2^{n}-1\right)$ where $S(0,0)=S, S(n+1, m)$ is the initial third of $S(n, m)$, and $S\left(n+1, m+2^{n-1}\right)$ is the terminal third of $S(n, m)$. For each nonnegative integer $n$ permute the segments $S(n, m)\left(0 \leqq m \leqq 2^{n-1}\right)$ cyclicly according to the second coordinate $m$. These permutations induce a homeomorphism $\phi$ of $X$ onto itself. More exactly, if $x \in X$, then for each nonnegative integer $n$ we have that $x \in S\left(n, m_{n}\right)$ for exactly one integer $m_{n}$ with $0 \leqq m_{n} \leqq 2^{n}-1$ and $x \phi=\bigcap_{n=0}^{+\infty} S\left(n, m_{n}+1\right.$ $\left.\left(\bmod 2^{n}\right)\right)$. It follows that $(X, \phi)$ is a regularly almost periodic mini- 
mal set which we may call the dyadic minimal set. To see the group structure of $X$, draw the horizontal segments $S(1,0)$ and $S(1,1)$ under the horizontal segment $S(0,0)$, draw downward arrows to $S(1,0)$ and $S(1,1)$ from $S(0,0)$, and label the arrows 0 and 1 from left to right. Continue this process. A point of $X$ is uniquely represented by a downward chain of arrows and therefore by a sequence of 0 's and 1's. The sequences are added coordinatewise with (possibly infinite) carry-over. The generator of the dyadic group $X$ which corresponds to $\phi$ is given by the sequence $(1,0,0,0, \cdots)$. The $n$-adic minimal sets are all regularly almost periodic.

In the case of the circle (1-sphere) as a minimal set under a discrete flow, the isomorphism type is characterized by an irrational number $r$ between 0 and 1 , the Poincare rotation number, and the minimal set is isomorphic to the spin of a circle through an angle which is $r \pi$ [for example, 17]. Actually, the discrete flows on a circle appear to be presently unclassified. Let us look at one. Let $\cdots, E_{-1}, E_{0}, E_{1}, \ldots$ be a disjoint bisequence of closed arcs on a circle $K$ such that $\bigcup_{n \in \mathcal{g}} E_{n}$ is dense in $K$ and such that the cyclic order of $\cdots, E_{-1}, E_{0}, E_{1}, \cdots$ on $K$ agrees with the cyclic order of $\cdots, x r^{-1}, x, x r, \cdots$ on a circle when the circle is rotated through one radian, $x$ being a point of the circle and $r$ denoting the rotation. Map $E_{n}(n \in \mathfrak{g})$ homeomorphically onto $E_{n+1}$ in the evident manner. This defines a homeomorphism of $\bigcup_{n \in \mathcal{g}} E_{n}$ onto itself. Since both this homomorphism and its inverse are uniformly continuous, it can be extended uniquely to a homeomorphism $\phi$ of $K$ onto $K$. The complement of $U_{n \in \mathcal{g}}$ int $E_{n}$ is a minimal set under $\phi$ which is the Cantor discontinuum and which is not almost periodic. The endpoints of the $E_{n}(n \in \mathcal{g})$ constitute a pair of doubly asymptotic orbits, that is $\rho\left(x \phi^{n}, y \phi^{n}\right)$ goes to zero with $1 / n$ where $x$ and $y$ are the endpoints of the arc $E_{0}$.

The almost periodic minimal sets are homogeneous since their phase spaces are groups. Is every minimal set homogeneous? The answer is no (Floyd 1949) [11]. Floyd's example may be described in geometric language (University of Pennsylvania dissertation of Joseph Auslander 1957) [3].

If $E$ is a box $A \times B$ where $A$ and $B$ are segments, define $E^{*}$ to be $\left(A_{1} \times B_{1}\right) \cup\left(A_{3} \times B\right) \cup\left(A_{5} \times B_{2}\right)$ where $A_{1}, \cdots, A_{5}$ are the consecutive equal fifths of $A$ and $B_{1}, B_{2}$ are the consecutive equal halves of $B$. If $D$ is a disjoint union of boxes, define $D^{*}$ to be the union of the $E^{*}$ where $E$ ranges over the boxes in $D$. Start with the unit box $X_{0}$ and define

$$
X_{n+1}=X_{n}^{*}(n=0,1,2, \cdots), \quad X=\bigcap_{n=0}^{+\infty} X_{n} .
$$


The boxes in $X_{n}(n=0,1,2, \cdots)$ are permuted just like the segments are permuted in the construction of the triadic minimal set. For example, the first three stages have boxes numbered as follows from left to right: $0 ; 0,1,2: 0,3,6,1,4,7,3,5,8$. The process gives rise to a homeomorphism $\phi$ of $X$ onto $X$. It is seen that $X$ consists of pointcomponents and segments, $X$ is zero-dimensional at point-components, $X$ is one-dimensional on segments, $X$ is therefore not homogeneous, $X$ is minimal under $\phi, \phi$ is not almost periodic, $\phi$ is regularly almost periodic at some points of $X$ but not at all points, $\phi$ is not recurrent and no orbits are unilaterally asymptotic.

There is also an example (F. B. Jones 1949) [14, p. 139 f.] of a minimal set under a discrete flow whose phase space is a plane onedimensional continuum which is locally connected at some points and not locally connected at other points.

We turn now to another class of examples which we shall call shifting flows. Consider a nonvacuous compact metric space $E$ and form its cartesian power $X=E^{\mathscr{I}}$ with exponent $\mathscr{I}$ where $\mathscr{g}$ is the set of all integers. An element of $X$ is simply a function on $\mathscr{g}$ to $E$. Provide $X$ with its product topology, or equivalently, its point-open topology. Define a homeomorphism $\sigma$ of $X$ onto $X$ as follows: $\left(x_{n} \mid n \in \mathfrak{g}\right) \sigma$ $=\left(x_{n+1} \mid n \in \mathfrak{g}\right)$ where $x=\left(x_{n} \mid n \in \mathfrak{g}\right) \in X$. The map $\sigma$ is cutomarily called the shift transformation. A point $x$ of $X$ may be denoted by a so-called symbolic trajectory.

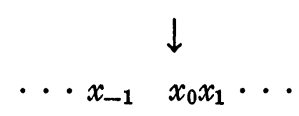

with index (the arrow) which denotes the value of the point $x$ at 0 . The shift transformation changes the indexed symbolic trajectory simply by shifting the index to the next symbol on the right. Thus

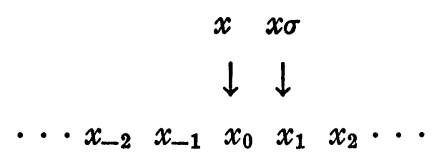

The notational device of a symbolic trajectory helps very considerably in the study of shifting flows.

A symbolic flow is defined to be a shifting flow $\left(E^{\mathfrak{I}}, \sigma\right)$ where $E$ is a finite set with more than one element. The phase space $E^{\mathfrak{g}}$ of a symbolic flow $\left(E^{\mathfrak{G}}, \sigma\right)$ is the Cantor discontinuum. Symbolic dynamics is defined to be the study of symbolic flows; it is therefore largely combinatorial in nature. A large number of papers have been concerned with symbolic dynamics $[14$, p. 113]. The symbolic flows are descrip- 
tive of geodesic flows over certain surfaces of constant negative curvature. Marston Morse (1921) [20] defined a symbolic trajectory as follows, where $E=\{a, b\}$ with $a \neq b$. The elements $a$ and $b$ are called duals of each other. Consider the 1-block $a$; dualize to form the 1-block $b$ and suffix it; we now have a 2-block $a b$. Consider the 2block $a b$; dualize to form the 2-block $b a$ and suffix it; we now have a 4-block $a b b a$. The next step gives the 8-block $a b b a b a a b$. The process is continued to form a ray. This ray is then reflected to the left to form a trajectory which may be indexed at any symbol. This indexed trajectory is an example of an almost periodic point under $\sigma$ which is not periodic. Consequently, Morse showed for the first time that there exist everywhere nonlocally connected compact minimal sets under certain geodesic flows.

A characteristic property of symbolic flows is expansive. A discrete flow $(X, \phi)$ is said to be expansive provided there exists a positive real number $\epsilon$ such that if $x$ and $y$ are different points of the phase space $X$, then $\rho\left(x \phi^{n}, y \phi^{n}\right)>\epsilon$ for some integer $n$. It may be seen that every symbolic flow is expansive and, conversely, if a discrete flow on a zero-dimensional compact metric space is expansive, then it may be imbedded in a symbolic flow. Observe that expansive is a strong negation of isometric, or equivalently, almost periodic. Consequently, the expansive minimal sets appear to be at the other end of the spectrum from almost periodic minimal sets. It has been proved (Schwartzmann 1952; Utz 1950 related) [23; 26; 14, p. 87] that every expansive discrete flow possesses a pair of points which is positively asymptotic and a pair of points which is negatively asymptotic. This means that $x \neq y$ and $\rho\left(x \phi^{n}, y \phi^{n}\right) \rightarrow 0$ as $n \rightarrow+\infty$ or $n \rightarrow-\infty$, respectively. The asymptotic points of a minimal set are at least partially characteristic of the minimal set.

In the shifting flow $\left(E^{\mathscr{G}}, \sigma\right)$ we may take $E$ to be the closed unit interval $J$ and obtain the Hilbert cube $J^{\mathscr{g}}$ as phase space. We may also take $E$ to be the circle group $K$ and obtain the infinite toral group $K^{\mathscr{I}}$ as phase space; in this case the shift transformation is a group automorphism. An example (R. F. Williams 1955) [28] of an expansive discrete flow on a continuum may be imbedded in the flow $\left(K^{\mathfrak{g}}, \sigma\right)$. It is described as the set of all bisequences $\left(z_{n} / n \in \mathfrak{g}\right)$ such that $z_{n}=z_{n+1}^{2}$ for all $n \in \mathfrak{g}$; it is therefore the 2-solenoidal group and the map is a group automorphism. The shifting flows in the Hilbert cube, the infinite torus, and other spaces, appear to be worthy of further study. So far as I am aware, shifting flows have been systematically investigated only in the case of symbolic flows.

I wish now to describe briefly some recent unpublished work [3, 
related] tending toward the classification of locally almost periodic minimal sets. Given a discrete flow $(X, \phi)$. The map $\phi$ is said to be locally almost periodic provided that if $x \in X$ and if $U$ is a neighborhood of $x$, then there exists a neighborhood $V$ of $x$ and a relatively dense subset $A$ of $g$ such that $V \phi^{n} \subset U$ for all $n \in A$. Two points $x$ and $y$ of $X$ are said to be proximal provided that if $\epsilon>0$, then $\rho\left(x \phi^{n}, y \phi^{n}\right)<\epsilon$ for some $n \in g$. Assume now that $X$ is compact metric and $\phi$ is locally almost periodic. The relation proximal is a closed equivalence relation in $X$. Consider now the star-closed partition space $X^{*}$ determined by the relation proximal. The discrete flow induced on $X^{*}$ is almost periodic. Suppose also that $X$ is minimal. Then $X^{*}$ is minimal. Hence $X^{*}$ is an almost periodic minimal set and thus a monothetic group, called the structure group of the original locally almost periodic minimal set. To illustrate, the structure group of Floyd's example is the triadic group, and the structure group of Jones' example in the circle group.

Just a few words now about continuous flows. In general, the definitions and theorems mentioned before apply to continuous flows as well as discrete flows. There are some exceptions, however. A minimal set under a continuous flow is necessarily connected; not so for a discrete flow. Another exception appears in the theorem (A. A. Markov 1931) $[19 ; 14$, p. 14] that a finite-dimensional minimal set under a continuous flow is a Cantor-manifold and hence has the same dimension at each point. This is not true for discrete flows: witness the example of Floyd.

The examples of continuous flows which have been most extensively studied are the geodesic flows over surfaces of constant negative curvature. Geodesic flows are defined for Riemannian manifolds of arbitrary dimension but let us think of the 2-dimensional case. Let $S$ be a surface of class $C^{2}$ provided with a Riemannian metric of class $C^{2}$ and which is complete in the sense that each geodesic arc in $S$ can be extended to infinite length in both directions. The phase space $X$ consists of the unit contravariant vectors on $S$ which we may call the unitangents (unitangent $=$ unit tangent). Let $x \in X$ and let $t \in R$. Consider the geodesic $g$ in $S$ which has $x$ as a tangent. If $t$ is positive, measure $t$ units of arc length along $g$ in the same direction as $x$ from the base point of $x$ and take the unitangent to $g$ at the new point to be the image of $x$ under the transition $\pi^{t}$. Likewise for negative $t$. There results a continuous flow which is called the geodesic flow over $S$. Now $X$ is a 3-dimensional manifold which is the bundle space of a fibre bundle with the circle as fiber and the surface $S$ as base space. The geodesic flows are dynamical systems which are determined by the Euler equations. 
We state one theorem on geodesic flows which is now classical. If $S$ is a complete 2-dimensional analytic Riemannian manifold of constant negative curvature and of finite area, then the geodesic flow over $S$ is regionally mixing and its periodic points are dense in the space of unitangents on $S$. This theorem is proved again in the Colloquium volume Topological dynamics [14, p. 131]. A continuous flow $(X, \Re, \pi)$ is said to be regionally mixing provided that if $U$ and $V$ are nonvacuous open subsets of $X$, then there exists a positive real number $s$ such that $U t \cap V \neq \varnothing$ for $t \in R$ with $|t| \geqq s$.

Closely related to the geodesic flows are the horocycle flows over surfaces of constant negative curvature. They have the same phase spaces as geodesic flows but different transitions and motions. It has been shown (Hedlund 1936) [16] that for each integer $p>1$ there exists a closed orientable surface $S$ of constant negative curvature and of genus $p$ such that the space $X$ of unitangents on $S$ is a regionally mixing minimal set under the horocycle flow over $S$. Here the phase spaces $X$ are compact 3-dimensional manifolds, are not almost periodic under the horocycle flows, and, even more, are not recurrent (since regionally mixing). A continuous flow $(X, R, \pi)$ is said to be recurrent provided that if $\epsilon>0$, then there exists an extensive subset $A$ of $R$ such that $x t \in N_{\epsilon}(x)$ for all $x \in X$ and all $t \in A$.

As remarked previously, every discrete flow $(X, \phi)$ gives rise to a continuous flow as follows: take the cartesian product of $X$ and the closed unit interval, and identify bases according to the map $\phi$. The capped $n$-adic minimal sets produce the $n$-solenoidal minimal sets. All of these minimal sets are regularly almost periodic, and their structure groups are the $n$-adic groups and the $n$-solenoidal groups.

Consider the space $X$ of all continuous functions $f$ on the line group $R$ to $R$ and provide $X$ with its compact-open topology. Define a continuous flow in $X$ by translation of the function $f:(f(r) \mid r \in R)$ $\rightarrow(f(r+t) \mid r \in R)$ defines $\pi^{t}$ for each $t \in R$. It is known (Bebutov 1940) [4] that every continuous flow in a compact metric space with at most one fixed point is imbeddable in the above flow. The minimal sets under continuous flows therefore all appear in the Bebutov example. A perspicuous proof of his theorem is much to be desired. From a topological point of view, the Bebutov example has a favorable property, namely, the orbit-closure of a point $f$ is compact if and only if $f$ is bounded and uniformly continuous.

The consideration of function spaces leads us to the almost periodic functions of Harold Bohr. Consider the space $X$ of all bounded uniformly continuous functions on $R$ to $R$ and provide $X$ with its uniform topology. Define a continuous flow in $X$ by translation of the functions. An element of $X$ is an almost periodic function in the sense 
of Bohr if and only if it is an almost periodic point under the flow. This statement shows the consistency of the terminology almost periodic. A certain fraction of the theory of almost periodic functions can be presented under the theory of continuous flows or, more generally, transformation groups, [14, pp. 40-48]. It seems that future developments will encompass more. Recent work of Tornehave (1954) [25] is suggestive in this connection.

In the foregoing discussion of flows there are several features which suggest a broader and more general development in the context of transformation groups. Some of these features are: (1) analogous theories for discrete flows and continuous flows (the famous dictum of $\mathrm{E}$. H. Moore is brought to mind); (2) the study of flows themselves leads to topological groups; (3) the study of flows leads to almost periodic functions and the theory of almost periodic functions includes functions on groups.

The Colloquium volume Topological dynamics [14] is written from this more general point of view. Let us mention briefly two representative notions of recursion for transformation groups. Let $(X, T)$ be a transformation group. A subset $A$ of the phase group $T$ is said to be syndetic provided that $T=A K$ for some compact subset $K$ of $T$. The notion syndetic replaces the notion relatively dense for sets in $\mathscr{I}$ and $\mathcal{R}$. A point $x$ of the phase space $X$ is said to be almost periodic provided that if $U$ is a neighborhood of $x$, then $x A=\{x t \mid t \in A\} \subset U$ for some syndetic subset $A$ of $T$. A semigroup $P$ in $T$ is said to be replete provided that $P$ contains some bilateral translate of each compact subset of $T$. A subset $A$ of $T$ is said to be extensive provided that $A$ intersects every replete semigroup in $T$. A point $x$ of $X$ is said to be recurrent provided that if $U$ is a neighborhood of $x$, then $x A \subset U$ for some extensive subset $A$ of $T$. When $T$ is $\mathscr{g}$ or $R$, then these notions of recursion reduce to the customary ones for flows [14].

For the reamining moments permit me to pose a few more questions which are presently unanswered so far as I know. Questions stated for flows on metric spaces are usually meaningful also for transformation groups on more general spaces.

(1) What compact metric spaces can be minimal sets under a discrete flow? Under a continuous flow? The universal curve of Sierpinski? The universal curve of Menger? A lens space? What polyhedra? Can they be nonorientable? About all that is known for polyhedra is that, in the continuous flow case, the Euler characteristic has to vanish. This follows from the fact that a minimal set with more than one point cannot have the fixed point property [1, p. 532]. The only closed surfaces with vanishing Euler characteristic are the torus 
and the Klein bottle. The torus is a minimal set under both discrete flows and continuous flows. Can the Klein bottle be a minimal set? What can be said about the homology and homotopy groups of a minimal set?

(2) More generally, what subsets of a given phase space can be minimal subsets? Does there exist a discrete flow in euclidean 3-space such that some orbit-closure is the necklace of Antoine? If so, can it be minimal? In an $n$-dimensional manifold it is known that a minimal subset is either the whole manifold or of dimension less than $n$ [14, p. 14]. If the dimension is equal to $n-1$, then is it necessarily an (n-1)-torus? (Question of R. W. Bass.) Bass has pointed out that a theorem of Kodaira and Abe (1940) [18] shows the answer to be affirmative for an almost periodic minimal set under a continuous flow even when merely the phase space is imbeddable in euclidean $n$-space.

(3) Let $X$ be a compact uniform space which is minimal under a homeomorphism $\phi$. In general, $X$ need not be metrizable since every compact connected separable abelian group is monothetic (for example, $K^{J}$ where $K$ is the circle group and $J$ is the unit interval). What other conditions will guarantee that $X$ be metrizable? One instance is that $\phi$ be expansive (Bryant 1955) $[7 ; 9]$. Another is that $\phi$ be regularly almost periodic.

(4) Distal means without distinct proximal points. Is every distal minimal set necessarily almost periodic? It is known that distal and locally almost periodic are equivalent to almost periodic, even without minimality [13].

(5) The study of geodesic flows and horocycle flows now requires rather much geometry and analysis. Is it possible, in some sense, to axiomatize these flows so that they are more accessible to immediate study?

(6) In many examples of geodesic flows, symbolic flows, and horocycle flows the following properties occur together: regionally mixing and dense periodic or almost periodic points. Does regionally mixing plus some auxiliary hypothesis imply that the almost periodic points are dense?

(7) What spaces can carry an expansive homeomorphism (Question of Utz and others)? The $n$-cells? May such spaces be locally connected?

(8) The roles of connected, locally connected, locally euclidean, and other kinds of connectivity, in question of existence and implication?

(9) When von Neumann defined his almost periodic functions on a 
group, he generalized Bochner's characterization of the Bohr almost periodic functions. This led to the notion of left and right almost periodic functions which Maak subsequently proved equivalent. (Incidentally, the theorems of topological dynamics can be quoted to establish this.) When the original Bohr definition is generalized directly to functions on topological groups, a similar dichotomy occurs but the answer as to their equivalence in this case is not clear. If the two kinds are actually equivalent, then this new definition leads again to von Neumann's functions. (See [14, p. 40 f.] for references and full statements.)

(10) There are a number of theorems in topological dynamics whose hypothesis and conclusion are meaningful for nonmetric spaces, say compact or locally compact uniform spaces, and yet the only known proof makes use of a category argument valid for metric spaces or at least first-countable spaces [14]. The question is to find a replacement for the category arguments, either in particular cases or in general, which permit the removal of countability assumptions such as metric or first-countable. Recent work of Robert Ellis (1957) $[10]$ is very interesting and suggestive in this connection.

(11) From an abstract point of view, there is an equivalence between transformation groups and spaces of functions on groups to spaces. Briefly, the space $X$ of functions on a group $T$ to a space $Y$ determines a transformation group $(X, T)$ by translation on the functions; and a transformation group $(X, T, \pi)$ determines the space $\left\{\pi_{x} \mid x \in X\right\}$ of motions in the space $Z$ of all functions on $T$ to $X$. These two constructions are not symmetric because the first produces a relatively complicated space $X$ from a relatively simple space $Y$, and the second produces a more complicated space $Z$ from an already complicated space $X$. To avoid the difficulty, a theorem is needed for transformation groups which extends the Bebutov theorem on a universal flow for continuous flows.

More questions are stated in [21].

\section{REFERENCES}

1. Paul Alexandroff and Heinz Hopf, Topologie I, Die Grundlehren der mathematischen Wissenschaften, vol. 45, Berlin, 1935; Ann Arbor, 1945.

2. Hirotada Anzai and S. Kakutani, Bohr compactifications of a locally compact abelian group II, Proceedings of the Imperial Academy, Tokyo, vol. 19 (1943) pp. 533-539.

3. Joseph Auslander, Mean-L-stable systems, Dissertation, University of Pennsylvania, 1957.

4. M. Bebutov, Sur les systèmes dynamiques dans l'espace des fonctions continues, Comptes Rendus, Doklady Akad. Nauk SSSR, vol. 27 (1940) pp. 904-906.

5. G. D. Birkhoff, Dynamical systems, Amer. Math. Soc. Colloquium Publications, vol. 9, New York, 1927. 
6. - Collected mathematical papers, 3 vols., American Mathematical Society, New York, 1950.

7. B. F. Bryant, Unstable self-homeomorphisms of a compact space, Dissertation, Vanderbilt University, 1954.

8. - A note on unstable homeomorphisms, Amer. Math. Monthly vol. 61 (1954) p. 509 (abstract).

9. - A note on uniform spaces, Amer. Math. Monthly, vol. 62 (1955) p. 529 (abstract).

10. Robert Ellis, Locally compact transformation groups, Duke Math. J. vol. 24 (1957) pp. 119-126.

11. E. E. Floyd, $A$ nonhomogeneous minimal set, Bull. Amer. Math. Soc. vol. 55 (1949) pp. 957-960.

12. W. H. Gottschalk, Almost periodic points with respect to transformation semigroups, Ann. of Math. vol. 47 (1946) pp. 762-766.

13. - Characterizations of almost periodic transformation groups, Proc. Amer. Math. Soc. vol. 7 (1956) pp. 709-712.

14. W. H. Gottschalk and G. A. Hedlund, Topological dynamics, Amer. Math. Soc. Colloquium Publications, vol. 36, Providence, 1955.

15. P. R. Halmos and H. Samelson, On monothetic groups, Proc. Nat. Acad. Sci. U.S.A., vol. 28 (1942) pp. 254-258.

16. G. A. Hedlund, Fuchsian groups and transitive horocycles, Duke Math. J. vol. 2 (1936) pp. 530-542.

17. E. R. van Kampen, The topological transformations of a simple closed curve into itself, Amer. J. Math. vol. 57 (1935) pp. 142-152.

18. Kunihiko Kodaira and Makoto Abe, Uber zusammenhängende kompakte abelsche Gruppen, Proceedings of the Imperial Academy, Tokyo, vol. 16 (1940) pp. 167-172.

19. A. A. Markoff, Sur une propriêtê générale des ensembles minimaux de M. Birkhoff, C. R. Acad. Sci. Paris vol. 193 (1931) pp. 823-825.

20. Marston Morse, Recurrent geodesics on a surface of negative curvature, Trans. Amer. Math. Soc. vol. 22 (1921) pp. 84-100.

21. V. V. Nemycki1, Topological problems of the theory of dynamical systems, Uspehi Mat. Nauk (N.S.) vol. 4 (1949) pp. 91-152 (Russian); Amer. Math. Soc. Translation, number 103.

22. V. V. Nemyckil and V. V. Stepanov, Qualitative theory of differential equations, Moscow-Leningrad, 1947; 2d ed., 1949, Russian; English translation of 2d ed., Princeton University Press, to appear in 1959. 1952.

23. Sol Schwartzman, On transformation groups, Dissertation, Yale University,

24. P. A. Smith, Periodic and nearly periodic transformations, Lectures in Topology, Ann Arbor, 1941, pp. 159-190.

25. Hans Tornehave, On almost periodic movements, Det Kongelige Danske Videnskabernes Selskab, Matematisk-fysiske Meddelelser, vol. 28, no. 13, 1954.

26. W. R. Utz, Unstable homeomorphisms, Proc. Amer. Math. Soc. vol. 1 (1950) pp. 769-774.

27. G. T. Whyburn, Analytic topology, Amer. Math. Soc. Colloquium Publications, vol. 28, New York, 1942.

28. R. F. Williams, A note on unstable homeomorphisms, Proc. Amer. Math. Soc. vol. 6 (1955) pp. 308-309.

\section{University of Pennsylvania}

